\title{
Comparison of perioperative characteristics and prognostic performance in patients with pulmonary lobectomy in early or later period after percutaneous coronary intervention
}

\author{
Tianyang Yun ${ }^{1,2}$, Yue Tang ${ }^{2}$, Bo Yang ${ }^{1}, \mathrm{Ke} \mathrm{Li}^{3}$, Tao Liang ${ }^{1}$, Dongtao Yin ${ }^{1}$, Yang Liu ${ }^{1}$ \\ ${ }^{1}$ Department of Thoracic Surgery, Chinese People's Liberation Army General Hospital, Beijing 100853, China; ${ }^{2}$ Department of Thoracic Surgery, \\ ${ }^{3}$ Department of Cardiology, Hainan Branch of Chinese People's Liberation Army General Hospital, Sanya 572013, China \\ Contributions: (I) Conception and design: T Yun, Y Liu; (II) Administrative support: None; (III) Provision of study materials or patients: T Yun, Y \\ Tang, B Yang, Y Liu; (IV) Collection and assembly of data: T Yun, T Liang, D Yin, Y Liu; (V) Data analysis and interpretation: T Yun, K Li, Y Liu; (VI) \\ Manuscript writing: All authors; (VII) Final approval of manuscript: All authors. \\ Correspondence to: Yang Liu. Department of Thoracic Surgery, Chinese People's Liberation Army General Hospital, Fuxing Road 28, Haidian \\ District, Beijing 100853, China. Email: sunny301x@sina.com.
}

\begin{abstract}
Background: In order to analyze the feasibility of pulmonary lobectomy for non-small cell lung cancer (NSCLC) in early period after percutaneous coronary intervention (PCI), the current study was designed to compare perioperative characteristics and prognostic performance of patients with pulmonary lobectomy within 3 months or 3 months later after PCI.

Methods: This study enrolled 349 patients simultaneously with NSCLC and coronary stenosis. There were 198 and 151 patients with pulmonary lobectomy within 3 months or 3 months later after PCI, respectively.

Results: Age of all patients was 62 [53-75] years. There was no difference in demographic characteristics, medical histories, cancer locations and stent numbers between two groups ( $\mathrm{P}>0.05$ for all). Operation time, blood loss and hospital stay after pulmonary lobectomy had no difference between two groups $(\mathrm{P}>0.05$ for all). Compared with those with pulmonary lobectomy 3 months later after PCI, survival rate during 5 years after pulmonary lobectomy was significantly higher in patients with pulmonary lobectomy within 3 months after PCI $(\mathrm{P}<0.05$ for all).

Conclusions: Patients with pulmonary lobectomy within 3 months after PCI had similar perioperative characteristics and better prognostic performance, as compared to those with pulmonary lobectomy 3 months later after PCI. The current study could provide valuable information in patients simultaneously with NSCLC and coronary stenosis to decide the timing of pulmonary lobectomy, and it might be feasible to perform pulmonary lobectomy in early period after PCI.
\end{abstract}

Keywords: Percutaneous coronary intervention (PCI); coronary stenosis; non-small cell lung cancer (NSCLC); pulmonary lobectomy

Submitted Jul 07, 2019. Accepted for publication Aug 28, 2019.

doi: $10.21037 /$ tcr.2019.09.23

View this article at: http://dx.doi.org/10.21037/tcr.2019.09.23

\section{Introduction}

Both non-small cell lung cancer (NSCLC) and coronary artery disease (CAD) have high incidence and mortality, and cause social and economic burden all over the world (1). Previous study has suggested that $26.5 \%$ of patients diagnosed with lung cancer also had different degrees of CAD (2). Moreover, as the most effective treatment of NSCLC, pulmonary lobectomy leads to a deadly cardiovascular risk (3). During perioperative period of pulmonary lobectomy, cardiovascular risk has been estimated to be as high as $4.2 \%$ (4). In patients with lung cancer combined with $\mathrm{CAD}$, percutaneous 
coronary intervention (PCI) has been considered as the optimal treatment to lower cardiovascular risk (5). Lacking antithrombotic therapy after PCI should be responsible for increased stent thrombosis and cardiovascular risk $(6,7)$. Based on the recommendation by general guidelines, noncardiac surgery is not very appropriate for patients who received PCI within 3-6 months (8).

However, it is not a good choice for patients with NSCLC to wait for delaying pulmonary lobectomy due to antithrombotic therapy after PCI. NSCLC cells with distinct differentiation degrees and histological types have a doubling time varying from 33 to 183 days, and its area could increase up to $373 \%$ after a median time of 54 days (9). Previous literature has shown that $26 \%$ of patients with lung cancer lost the opportunity to receive radical resection due to antithrombotic therapy (10). Simultaneous coronary artery bypass grafting and pulmonary lobectomy in patients with NSCLC have been tried previously, with a result of prolonged time of operation and hospitalization, as well as increased incidence of postoperative complications (11-13). The issue on how to perform pulmonary lobectomy as soon as possible without increasing cardiovascular risk is of great importance and needs to be clarified. In order to analyze the feasibility of pulmonary lobectomy for NSCLC in early period after PCI, the current study was designed to compare perioperative characteristics and prognostic performance of patients with pulmonary lobectomy within 3 months or 3 months later after PCI.

\section{Methods}

\section{Study population}

From January 2006 to December 2012, this retrospective study analyzed 562 patients who simultaneously had lung cancer and coronary stenosis in Chinese People's Liberation Army Hospital. Chest-enhanced computed tomography revealed that all of them had a diagnosis of lung cancer, and then 65 patients were excluded due to multiple pulmonary lobes or severe cancer metastasis found in examinations and no need of pulmonary lobectomy. A diagnosis of lung cancer was excluded in 12 patients due to pathological examination during operation. All patients with symptoms of myocardial ischemia received computed tomography angiography, which revealed that they had coronary stenosis and required coronary angiography. There were 123 patients excluded due to no severe coronary stenosis found in coronary angiography and no need of PCI, and
13 patients excluded due to small cell lung cancer and no need of pulmonary lobectomy. In all, 349 patients simultaneously received the treatment of pulmonary lobectomy and PCI. There were 198 and 151 patients who received pulmonary lobectomy within 3 months or 3 months later after PCI, respectively.

\section{Study procedure}

Demographic characteristics and medical histories were obtained in all patients. Chest-enhanced computed tomography and computed tomography angiography were performed to assess lesion condition and severity. Cardiac and pulmonary function was assessed by echocardiogram and respiratory function instrument. Patients with suspected central-type lung cancer also received electronic fibre bronchoscope. Brain computed tomography, radionuclide bone scanning and ultrasounds in abdomen and lymph nodes were performed to identify patients with severe cancer metastasis who did not require pulmonary lobectomy. Perioperative characteristics and prognostic performance were compared between patients with pulmonary lobectomy within 3 months or 3 months later after PCI. Survival rate was the primary end-point, and no one was lost, during 5 years after pulmonary lobectomy.

\section{Operative procedure}

Patients received coronary angiography under local anesthesia by one surgical team. Patients received stent implantation due to severe coronary stenosis. PCI had a success rate of $100 \%$. Patients received open and videoassisted thoracoscopic pulmonary lobectomy under double lumen endotracheal intubation and general anesthesia by one surgical team. Single lung ventilation was performed in health lung. The upper lobe was resected from the fourth intercostal space, and the middle and lower lobes were resected from the fifth intercostal space. Hilar and mediastinal lymph nodes were dissected as follows: group 2-4 and 7-10 (right chest) and group 4-10 (left chest). Specimen was submitted to Pathology Department in Chinese People's Liberation Army Hospital for pathological examination. Success rate of pulmonary lobectomy was $100 \%$, and there was no occurrence of pulmonary infection, wound infection, secondary operation and perioperative death. Postoperative chemotherapy was applied based on the TNM stages of patients as shown in Table 1. It was applied in patients with TNM stage II and TNM stage IIIa 
Table 1 Comparison of perioperative characteristics between patients with pulmonary lobectomy within 3 months or 3 months later after PCI

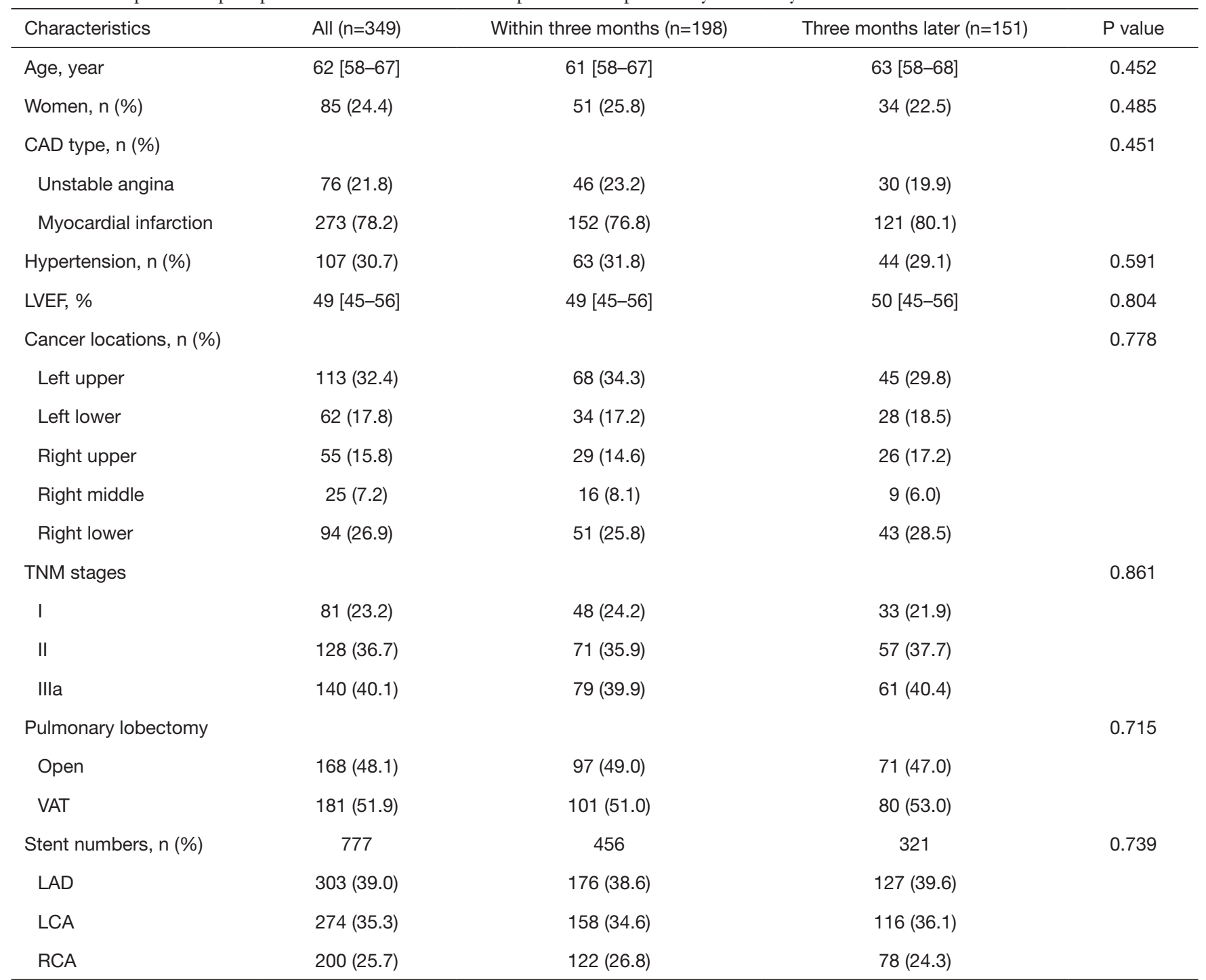

$\mathrm{PCl}$, percutaneous coronary intervention; CAD, coronary artery disease; LVEF, left ventricular ejection fraction; LAD, left anterior descending artery; LCA, left circumflex artery; RCA, right coronary artery; VAT, video-assisted thoracoscopic.

rather than TNM stage I.

\section{Antitbrombotic treatment}

Patients were administered loading doses $(300 \mathrm{mg})$ of aspirin and clopidogrel before PCI $(14,15)$. During PCI, patients were firstly administered unfractionated heparin $(3,000 \mathrm{U})$. An additional dose (50-100 U/kg) of unfractionated heparin was administered after determining the protocol of PCI (16). Tirofiban was administered (5-8 $\mathrm{mL} / \mathrm{h}$ ) for 3 days after PCI, and patients took a daily dose of aspirin $(100 \mathrm{mg})$ and clopidogrel $(75 \mathrm{mg})$. Aspirin and clopidogrel were discontinued 7 days before pulmonary lobectomy, and low molecular heparin was administered until 12 hours before pulmonary lobectomy. Tirofiban (5-8 $\mathrm{mL} / \mathrm{h})$ was administered 3 days after pulmonary lobectomy, lasting for 7-14 days or until hospital discharge. Patients took a daily dose of aspirin $(100 \mathrm{mg})$ and 
Table 2 Comparison of prognostic performance between patients with pulmonary lobectomy within 3 months or 3 months later after PCI

\begin{tabular}{|c|c|c|c|c|}
\hline Characteristics & All $(n=349)$ & Within three months $(n=198)$ & Three months later $(n=151)$ & $P$ value \\
\hline Blood loss, mL & $191[175-206]$ & 192 [176-208] & 189 [175-205] & 0.346 \\
\hline Chest drainage, $\mathrm{mL}$ & 718 [488-757] & $751[737-766]$ & 485 [468-501] & $<0.001$ \\
\hline Drainage time, $\mathrm{d}$ & $4[2-7]$ & $6[2-8]$ & $2[1-5]$ & $<0.001$ \\
\hline Survival rate, $\mathrm{n}(\%)^{\mathrm{a}}$ & $81(23.2)$ & $54(27.3)$ & 27 (17.9) & 0.039 \\
\hline
\end{tabular}

$\mathrm{PCl}$, percutaneous coronary intervention. ${ }^{\mathrm{a}}$, After pulmonary lobectomy.

clopidogrel (75 mg) after hospital discharge.

\section{Statistical analysis}

Continuous variables with normal distribution were described with mean (standard deviation), and compared between two groups with Student's $t$-test. Continuous variables with skewed distribution were described with median (interquartile range), and compared between two groups with Mann-Whitney U test. Categorical variables were described with number (percentage), and compared between two groups with Chi-square test. $\mathrm{P}$ value $<0.05$ was considered as statistically significant. Statistical analysis was performed by Statistic Package for Social Science (SPSS) version 17.0 software (SPSS Inc., Chicago, USA).

\section{Results}

Age of all patients was 62 [53-75] years, and $75.6 \%$ were men (264 patients). As shown in Table 1, there was no difference in demographic characteristics, medical histories, cancer locations, TNM stages and stent numbers between two groups ( $\mathrm{P}>0.05$ for all). Operation time, blood loss and hospital stay after pulmonary lobectomy had no difference between two groups ( $\mathrm{P}>0.05$ for all; Table 2). Chest drainage was significantly less and drainage time was significantly shorter in patients with pulmonary lobectomy 3 months later after PCI than those with pulmonary lobectomy within 3 months after PCI ( $\mathrm{P}<0.05$ for all). Compared with those with pulmonary lobectomy 3 months later after PCI, survival rate during 5 years after pulmonary lobectomy was significantly higher in patients with pulmonary lobectomy within 3 months after $\mathrm{PCI}(\mathrm{P}<0.05$ for all).

\section{Discussion}

In patients with NSCLC, pulmonary lobectomy is considered to be the most effective method with significant ability to improve their prognosis (3). Patients with pulmonary lobectomy often has different degrees of CAD and increased cardiovascular risk (2). Based on previous data, cardiovascular risk has been estimated to be as high as $4.2 \%$ in patients with pulmonary lobectomy (4). Antithrombotic therapy is crucial in patients with CAD to reduce stent thrombosis and cardiovascular risk $(6,7)$. There is a controversy about the timing of pulmonary lobectomy and the safety of antithrombotic therapy after PCI. Previous study has suggested that pulmonary lobectomy in early period after PCI might worsen the prognosis of patients with NSCLC (17). However, rapid growth of NSCLC cells was irreversible if not be resected, and could cause patients to lose their opportunity for timely treatment $(9,10)$. The current study compared perioperative characteristics and prognostic performance of patients with pulmonary lobectomy within 3 months or 3 months later after PCI to provide valuable information in clinical decision. Compared with those with pulmonary lobectomy 3 months later after PCI, the current study found similar perioperative characteristics and better prognostic performance in patients with pulmonary lobectomy within 3 months after PCI.

With regard to perioperative characteristics, patients with pulmonary lobectomy within 3 months after PCI had no obviously increased blood loss than those with pulmonary lobectomy 3 months later after PCI. The current study applied a clinically acceptable antithrombotic strategy, which might effectively balance perioperative hemorrhagic and thrombotic risk (14-16). As an antithrombotic drug 
with a short half-life period, tirofiban might be safe during perioperative period of pulmonary lobectomy after PCI. Chest drainage was significantly increased and drainage time was significantly lengthened by advanced pulmonary lobectomy. The dissociation of extensive adhesion aggravates pleural effusion, and antithrombotic therapy could further increase effusion (5).

Compared with those with pulmonary lobectomy 3 months later after PCI, these was no obviously prolonged operation time and hospital stay in patients with pulmonary lobectomy within 3 months after PCI. Operative complexity and patient recovery might not be affected by advanced pulmonary lobectomy. More importantly, survival rate during 5 years after pulmonary lobectomy was significantly improved due to advanced pulmonary lobectomy. Pulmonary lobectomy within 3 months after PCI combined with appropriate antithrombotic strategy might advance the timing of pulmonary lobectomy, improve the prognosis of patients with NSCLC and allow more patients to receive operative treatment.

\section{Conclusions}

The current study demonstrated similar perioperative characteristics and better prognostic performance in patients with pulmonary lobectomy within 3 months after PCI, as compared to those with pulmonary lobectomy 3 months later after PCI. The current study could provide valuable information in patients simultaneously with NSCLC and CAD to decide the timing of pulmonary lobectomy, and it might be feasible to perform pulmonary lobectomy in early period after PCI.

\section{Acknowledgments}

We are grateful to all study participants for their participation in the study.

Funding: None.

\section{Footnote}

Conflicts of Interest: All authors have completed the ICMJE uniform disclosure form (available at http://dx.doi. org/10.21037/tcr.2019.09.23). The authors have no conflicts of interest to declare.

Ethical Statement: The authors are accountable for all aspects of the work in ensuring that questions related to the accuracy or integrity of any part of the work are appropriately investigated and resolved. The study protocol has been approved by Ethics Committee of Chinese People's Liberation Army General Hospital (No: 2012031) and it conforms to the Helsinki Declaration. Patient's consent was waived in this retrospective study.

Open Access Statement: This is an Open Access article distributed in accordance with the Creative Commons Attribution-NonCommercial-NoDerivs 4.0 International License (CC BY-NC-ND 4.0), which permits the noncommercial replication and distribution of the article with the strict proviso that no changes or edits are made and the original work is properly cited (including links to both the formal publication through the relevant DOI and the license). See: https://creativecommons.org/licenses/by-nc-nd/4.0/.

\section{References}

1. Siegel RL, Miller KD, Jemal A. Cancer statistics, 2015. CA Cancer J Clin 2015;65:5-29.

2. Ohmoto-Sekine Y, Yanagibori R, Amakawa K, et al. Prevalence and distribution of coronary calcium in asymptomatic Japanese subjects in lung cancer screening computed tomography. J Cardiol 2016;67:449-54.

3. Patel RL, Townsend ER, Fountain SW. Elective pneumonectomy: factors associated with morbidity and operative mortality. Ann Thorac Surg 1992;54:84-8.

4. Brunelli A, Cassivi SD, Fibla J, et al. External validation of the recalibrated thoracic revised cardiac risk index for predicting the risk of major cardiac complications after lung resection. Ann Thorac Surg 2011;92:445-8.

5. Li X, Fu Y, Miao J, et al. Video-assisted thoracoscopic lobectomy after percutaneous coronary intervention in lung cancer patients with concomitant coronary heart disease. Thorac Cancer 2017;8:477-81.

6. Kałuza GL, Joseph J, Lee JR, et al. Catastrophic outcomes of noncardiac surgery soon after coronary stenting. J Am Coll Cardiol 2000;35:1288-94.

7. Vicenzi MN, Meislitzer T, Heitzinger B, et al. Coronary artery stenting and noncardiac surgery--a prospective outcome study. Br J Anaesth 2006;96:686-93.

8. Chatterjee A, Hage FG. Guidelines in review: 2014 ACC/ AHA guideline on perioperative cardiovascular evaluation and management of patients undergoing noncardiac surgery: A report of the American College of Cardiology/ American Heart Association Task Force on practice guidelines. J Nucl Cardiol 2015;22:158-61. 
9. O'Rourke N, Edwards R. Lung cancer treatment waiting times and tumour growth. Clin Oncol (R Coll Radiol) 2000;12:141-4.

10. Buccheri G, Ferrigno D. Lung cancer: Clinical presentation and specialist referral time. Eur Respir J 2004;24:898-904.

11. Ahmed AA, Sarsam MA. Off-pump combined coronary artery bypass grafting and left upper lobectomy through left posterolateral thoracotomy. Ann Thorac Surg 2001;71:2016-8.

12. Mariani MA, van Boven WJ, Duurkens VA, et al. Combined offpump coronary surgery and right lung resections through midline sternotomy. Ann Thorac Surg 2001;71:1343-4

13. Danton MHD, Anikin V, McManus KG, et al. Simultanous cardiac surgery with pulmonary resection: presentation of series and review of literature. Eur J Cardiothorac Surg 1998;13:667-72.

14. Di Sciascio G, Patti G, Pasceri V, et al. Effectiveness of inlaboratory high-dose clopidogrel loading versus routine preload in patients undergoing percutaneous coronary intervention: Results of the ARMYDA-5 PRELOAD (Antiplatelet therapy for reduction of MYocardial Damage during Angioplasty) randomized trial. J Am Coll Cardiol 2010;56:550-7.

15. Widimsky P, Motovská Z, Simek S, et al. Clopidogrel pretreatment in stable angina: For all patients $>6$ $\mathrm{h}$ before elective coronary angiography or only for angiographically selected patients a few minutes before PCI? A randomized multicentre trial PRAGUE-8. Eur Heart J 2008;29:1495-503.

16. Schulz S, Mehilli J, Neumann FJ, et al. ISAR-REACT 3A: A study of reduced dose of unfractionated heparin in biomarker negative patients undergoing percutaneous coronary intervention. Eur Heart J 2010;31:2482-91.

17. Cruden NL, Harding SA, Flapan AD, et al. Previous coronary stent implantation and cardiac events in patients undergoing noncardiac surgery. Circ Cardiovasc Interv 2010;3:236-42.

Cite this article as: Yun T, Tang Y, Yang B, Li K, Liang T, Yin D, Liu Y. Comparison of perioperative characteristics and prognostic performance in patients with pulmonary lobectomy in early or later period after percutaneous coronary intervention. Transl Cancer Res 2019;8(5):2073-2078. doi: $10.21037 /$ tcr.2019.09.23 